УДК 681.3 .08

\title{
Ю.Я. Самохвалов
}

\section{Оценка обоснованности управленческих решений на основе нечеткой логики}

\begin{abstract}
|Предложен подход к оценке обоснованности управленческих решений, согласно которому обоснованность решения рассматривается как степень истинности всех его положений. В основу подхода положен логико-лингвистический анализ факторов, определяющих обоснованность и нечеткий вывод, что позволяет получить доказательные оценки обоснованности принимаемых управленческих решений в различных сферах деятельности.

Ключевые слова: управленческое решение, качество и обоснованность решений, нечеткость, лингвистическая переменная, нечеткая логика, доказательство.

Запропоновано підхід до оцінки обгрунтованості управлінських рішень, згідно з яким обгрунтованість рішення розглядається як ступінь істинності всіх його положень. В основу підходу покладено логіко-лінгвістичний аналіз чинників, які визначають обгрунтованість і нечіткий вивід, що дозволяє отримати доказові оцінки обгрунтованості управлінських рішень, які приймаються в різних сферах діяльності.

Ключові слова: управлінське рішення, якість і обгрунтованість рішень, нечіткість, лінгвістична змінна, нечітка логіка, доведення.
\end{abstract}

Введение. В динамике любого управления тесно переплетаются интуитивные и научно обоснованные решения, т.е. фундамент, на который накладываются творческие замыслы человека. Существенное практическое значение приобретает оценка обоснованности в интеллектуальных системах поддержки принятия решений, в которых система формирует возможные варианты решений, а окончательное решение принимает человек и несет полную ответственность за последствия от его реализации.

Обоснованность определяет качество принимаемых решений, и измерить ее в процессе управления невозможно. О качестве принятых и реализованных решений, а следовательно, и об их обоснованности можно судить лишь по их последствиям. Поэтому априорная оценка обоснованности решений крайне необходима.

Существует несколько подходов к оценке обоснованности решений [1-3]: вариантный, оценка по критерию эффективности, вероятностный и информационный. В этих подходах обоснованность решений оценивается по отклонениям параметров управления от экстремальных значений, присущих оптимальным решениям, что требует знания такого решения. Кроме этого не в полном объеме учитываются факторы, определяющие обоснованность решений, что, в совокупности, не всегда обеспечивает достоверность таких оценок.

Оценка обоснованности решений - это предмет квалиметрии, одной из главных проблем которой является разработка механизмов преобразования параметров объекта в показатели его качества. Наиболее существенными факторами, определяющими обоснованность решений, являются достоверность и полнота исходных данных, а также качество модели выработки решения. Для эффективного решения этой проблемы могут быть использованы механизмы нечеткой логики, поскольку данные факторы имеют нечеткий характер. В настоящее время нечеткое моделирование является одной из наиболее активных и перспективных направлений прикладных исследований в области управления и оценки его качества $[4,5]$.

В статье предложен один из возможных подходов к оценке обоснованности решений, построенный на логико-лингвистическом анализе морфологии решений, факторов, определяющих их качество и влияние этих факторов на обоснованность решений.

\section{Структура управленческих решений}

Управленческое решение - выбор целенаправленного воздействия на объект управления, который базируется на анализе ситуации и со- 
держит программу достижения цели. Эти решения служат составной частью любой функции процесса управления и пронизывают всю управленческую деятельность - от формулировки цели до момента ее достижения путем реализации.

Любому действию, как правило, предшествуют анализ и оценка обстановки (ситуации), формирование плана действий и его осуществление. Оценка обстановки - первый этап подготовки определенного действия, который может быть и самостоятельной задачей. Оценить обстановку значит построить ее модель с определенной степенью детализации; установить существенные признаки обстановки и относительно каждого из них решить, существует ли он в данной ситуации. Результатом оценки обстановки будут $u н$ формационные решения, которые дают ответы на вопросы, определяющие цель предстоящих действий. Например, что, когда и где произошло? Любые просчеты в оценке обстановки могут привести к нежелательным последствиям - неоправданным временным и материальным потерям. Поэтому принято считать, что правильная оценка обстановки - уже половина решения поставленной задачи.

Затем принимаются организационные решения, которые определяют стратегию предстоящих действий и отвечают на вопрос: что необходимо предпринять в данной ситуации для достижения цели? И, наконец, принимаются оперативные решения, определяющие тактику действий. Эти решения отвечают на вопрос: как действовать, чтобы достичь цель? Рассмотренные решения выступают как этапы и элементы общего решения и составляют его содержание.

\section{Нечеткая обоснованность}

Обоснование решения - способ убеждения в его истинности (правильности), т.е. приведение тех убедительных аргументов или доводов, в силу которых следует принять решение. Это обоснование тесно связано с логикой, поэтому обоснованием решения служит логическое доказательство истинности всех его положений. Решение считается обоснованным, когда каждое его положение и/или параметр управления подтвержден объективными фактами реального мира и/ или закономерностями управляемого процесса.
Термин обоснованность носит «размытый» характер, поэтому его можно рассматривать как нечеткую переменную «обоснованно» с областью определения $[0,1]$. Заметим, что теоретически значение обоснованности никогда не может достигнуть единищьы. Поскольку при любых объеме и глубине обоснований всегда остается возможность учесть дополнительные данные и тем самым улучшить качество принимаемого решения.

Для задания нечеткой обоснованности будем исходить из следующих соображений. Обоснованность решения есть истинность его положений, поэтому для задания нечеткой обоснованности можно использовать известные функции принадлежности для нечеткой переменной $u c$ тинно. Так как рассматриваются значения истинности из интервала $[0,1]$, то наиболее интуитивно понятной будет функция принадлежности Балдвина:

$$
\mu \text { «обосновано» }(x)=x, x \in[0,1] .
$$

При оценке обоснованности решения как вероятности его истинности, следует исходить из того, что эта оценка имеет не статистический смысл, а смысл психологической уверенности. По мнению Д. Пойя, в таких случаях числовое выражение обоснованности не применимо, и следует использовать модальные категории такие, как средняя обоснованность, большая обоснованность и др.

Для оценки различных показателей качественного характера широкое распространение получила вербально-числовая шкала Харрингтона (табл. 1). Шкала универсальна, однако при

Та блли ц а 1

\begin{tabular}{|l|c|}
\hline \multicolumn{1}{|c|}{ Модальная оценка } & Числовое значение \\
\hline Очень высокая & $0,8-1,0$ \\
\hline Высокая & $0,64-0,8$ \\
\hline Средняя & $0,37-0,64$ \\
\hline Низкая & $0,2-0,37$ \\
\hline Очень низкая & $0,0-0,2$ \\
\hline
\end{tabular}

оценке обоснованности управленческих решений, особенно связанных с управлением объектами критической инфраструктуры, очевидно, не достаточно точна. Поэтому для оценки обоснованности управленческих решений целесо- 
образны разработка и использование специальных модификаций шкалы, отражающих специфику того или иного управленческого решения.

\section{Оценка обоснованности решений}

Обоснованность - качественная характеристика решений, поэтому для ее оценки будем использовать экспертные суждения, которые в предлагаемом подходе составляют информационный базис нечеткого вывода как инструмента получения такой оценки.

Основу нечеткого логического вывода составляет композиционное правило Заде, которое по отношению $\tilde{A} \rightarrow \tilde{B}$ между нечеткими высказываниями $\tilde{A}$ и $\tilde{B}$ и посылке $\tilde{A}$ позволяет получить истинность заключения $\tilde{B}$ по формуле

$$
\tilde{B}=\tilde{A} \bullet(\tilde{A} \rightarrow \tilde{B}),
$$

где «๑» - операция максиминной композиции.

Для оценки степени истинности нечетких высказываний из некоторого множества $\tilde{U}$ введем отображение $T$, которое ставит в соответствие каждому нечеткому высказыванию из $\tilde{U}$ степень его истинности из интервала $[0,1]$, т.е. $T: \tilde{U} \rightarrow[0,1]$. Это отображение будем называть отображением истинности нечетких высказываний, а значение истинности нечеткого высказывания $\tilde{C} \in \tilde{U}$ обозначим через $T(\tilde{C})$. Тогда (2) можно записать так:

$$
\begin{gathered}
T(\tilde{B})=\max (\min (T(\tilde{A}) ; T(\tilde{A} \rightarrow \tilde{B}))), \text { или } \\
T(\tilde{B})=\min (T(\tilde{A}) ; T(\tilde{A} \rightarrow \tilde{B}))
\end{gathered}
$$

для случая, когда вывод построен на единственном нечетком правиле $\tilde{A} \rightarrow \tilde{B}$.

При этом, если нечеткое высказывание $\tilde{A}$ является составным, то его истинность $T(\tilde{A})$ будем вычислять по классическим формулам min-конъюнкции и max-дизъюнкции. Заметим также, поскольку истинность $T(\tilde{A} \rightarrow \tilde{B})$ не может быть получена по известным формулам вычисления нечеткой импликации, так как они требуют знания значения $T(\tilde{B})$, то вместо классической импликации $\tilde{A} \rightarrow \tilde{B}$ используем предложенную Мамдани для систем нечеткого вывода импликацию вида $\tilde{A} \rightarrow \tilde{B}(F)$. Здесь
$F \in[0,1]$ - коэффициент уверенности, выражающий оценку значимости этой импликации, или уверенность в степени истинности ее заключения. По умолчанию коэффициент уверенности принимается равным единице. Этот коэффициент будет выступать в качестве значения $T(\tilde{A} \rightarrow \tilde{B})$.

Далее, любое управляющее решение, в общем случае, состоит из трех частных решений информационного, организационного и оперативного. Пусть $R\left(r_{1}, r_{2}, r_{3}\right)$ - управляющее решение, а $r_{i}$ - частные решения. Тогда наблюдается следующая зависимость:

$$
\left(\tilde{O}\left(r_{1}\right) \wedge \tilde{O}\left(r_{2}\right) \wedge \tilde{O}\left(r_{3}\right)\right) \rightarrow \tilde{O}(R),
$$

где $\tilde{O}(R)$ и $\tilde{O}\left(r_{i}\right)$ - нечеткие высказывания, обозначающие обоснованность решений $R$ и $r_{i}$ соответственно.

Поскольку рассматриваемый подход обладает достаточной общностью, рассмотрим оценки $\tilde{O}\left(r_{i}\right)$ на примере оценки обоснованности информационного решения.

Как было отмечено, обоснованность любого решения зависит от трех основных факторов: достоверности и полноты исходных данных и качества модели выработки решения, которая отражает глубину научного познания закономерностей управляемого процесса и степень использования этих знаний при обосновании конкретного решения.

Пусть $r$ - информационное решение, $D$ исходные данные, использованные при выработке этого решения, $M$ - модель выработки решения $r$. Далее, пусть $\tilde{A}(D), \tilde{P}(D), \tilde{K}(M)-$ нечеткие высказывания, обозначающие соответственно достоверность исходных данных $D$, их полноту и качество модели $M$. Тогда связь данных факторов с обоснованностью решения $r$ можно представить следующим нечетким продукционным правилом:

$$
(\tilde{A}(D) \wedge \tilde{P}(D) \wedge \tilde{K}(M)) \rightarrow \tilde{O}(r)
$$

и задачу оценки обоснованности решения $r$ свести к оценке истинности антецедента $(\tilde{A}(D), \tilde{P}(D), \tilde{K}(M))$ этого правила. 
Оценка достоверности исходных данных. Под достоверностью информации будем понимать ее свойство отражать объективную реальность с необходимой точностью. Критерий достоверной информации - отсутствие искажённых или ложных данных, а в качестве меры количественной оценки используется вероятность ее истинности.

Достоверность информации в общем зависит от двух факторов: достоверности (надежности) источника информации и способа получения информации. Источником информации при подготовке управляющих решений могут быть люди, документы и технические средства (системы) [6].

Надежность источника характеризуется его способностью выдачи истинных данных и определяется его характеристиками. Для технических средств такими характеристиками будут их технические параметры. Если источником информации служит человек, то помимо личных качеств, необходимо также учитывать его психофизиологическое состояние, от которого зависит уровень и качество восприятия окружающей обстановки. Критерии качества информации, по которым может быть осуществлена оценка надежности источника, приведены в [7].

При оценке достоверности информации также необходимо знать способ получения первичных данных источником, так как даже полная надежность источника не гарантируют ее достоверности. Так, сведения «из первых рук» достовернее информации из неопределенного источника, а записи по свежим впечатлениям отличаются от описания тех же событий спустя какое-то время. Отмечают следующие способы получения информации источником: она получена самостоятельно; из другого постоянного источника (например, открытого источника), которому можно верить, или из другого «разового» источника (в ходе переговоров, неформального общения и пр.).

Далее, пусть $\tilde{A}(S, D)$ и $\tilde{A}(Z, D)$ - нечеткие высказывания, обозначающие надежность источника $S$, выдавших данные $D$ и достоверность способа получения источником $S$ этих данных. Тогда формально связь данных факторов с достоверностью данных $D$ можно представить так:

$$
(\tilde{A}(S, D) \wedge \tilde{A}(Z, D)) \rightarrow \tilde{A}(D) .
$$

При оценке истинности высказываний $\tilde{A}(S, D)$ и $\tilde{A}(Z, D)$ будем использовать схему Кента $[8$, 9], которая дает наглядную классификацию информации по степени ее достоверности (рис. 1).

Пусть $D=\left\{d_{i} \mid i=\overline{1, n}\right\}$ - множество исходных данных, $S=\left\{s_{i} / z_{i}\right\}$ - множество источников, где $s_{i}$ - источник данных $d_{i}$, а $z_{i}$ - способ получения этих данных источником $s_{i}$. Предположим также, что для оценки достоверности данных $d_{i}$ проводится групповая экспертиза.

Далее, пусть $\xi_{j}^{i}\left(s_{i}\right)$ и $\xi_{j}^{i}\left(z_{i}\right)$ - соответственно оценки достоверности источника $s_{i}$ и способа $z_{i}$, получены $j$-м экспертом. Тогда его оценка достоверности данных $d_{i}$ вычисляется как $\xi_{j}^{i}\left(d_{i}\right)=\min \left(\xi_{j}^{i}\left(s_{i}\right), \xi_{j}^{i}\left(z_{i}\right)\right)$. Заметим, когда имеется $k$ источников данных $d_{i}$, оценка $\xi_{j}^{i}\left(d_{i}\right)$ получается в результате максиминной свертки $\xi_{j}^{i}\left(d_{i}\right)=\max _{k}\left(\min \left(\xi_{j}^{i}\left(s_{i}\right), \xi_{j}^{i}\left(z_{i}\right)\right)\right)$. В результате оценку достоверности (вероятности истинности) данных $d_{i}$, согласно [10], можно вычислить по формуле

$$
p\left(d_{i}\right)=\sum_{j=1}^{m} r_{j} \cdot \xi_{j}^{i}\left(d_{i}\right)
$$

где $m$ - количество экспертов, $r_{j}-$ их весовые коэффициенты, причем $\sum_{j=1}^{m} r_{j}=1$.

В итоге как достоверность данных $D$ берется минимальное из значений $p\left(d_{i}\right)$, которое будет значением истинности нечеткого высказывания $\tilde{A}(D)$, т.е. $T(\tilde{A}(D))=\min _{i}\left(p\left(d_{i}\right)\right)$.

Оценка полноты исходных данных. Неполнота данных связана с основным информационным диалектическим противоречием между необходимостью полного знания обстановки для принятия оптимального решения и 


\begin{tabular}{|c|c|c|c|c|c|}
\hline \multicolumn{6}{|c|}{ ДОСТОВЕРНОСТЬ } \\
\hline \multicolumn{2}{|c|}{$\begin{array}{c}\text { Шансы } \\
\text { за } \\
\end{array}$} & & $\begin{array}{l}\text { Шансы } \\
\text { против }\end{array}$ & $\begin{array}{c}\text { Степень достоверности, } \\
\text { в шансах }\end{array}$ & $\begin{array}{c}\text { Степень достоверности, выражен- } \\
\text { ная через понятия вероятности }\end{array}$ \\
\hline \multirow{5}{*}{ 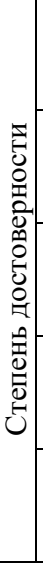 } & 99 & 888888 & 15 & $\begin{array}{l}\text { Почти определенно, инфор- } \\
\text { мация достоверная (за - 9, } \\
\text { против - 1) }\end{array}$ & $\begin{array}{l}\text { Почти определенно, информация } \\
\text { достоверная - да }\end{array}$ \\
\hline & 84 & I & 16 & $\begin{array}{l}\text { Имеется много шансов, что } \\
\text { информация достоверная } \\
(\text { за }-3, \text { против }-1)\end{array}$ & $\begin{array}{l}\text { Вероятно, информация достовер- } \\
\text { на - да }\end{array}$ \\
\hline & 59 & 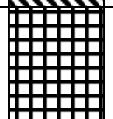 & 41 & $\begin{array}{l}\text { Шансы примерно равны } \\
(\text { за }-1, \text { против }-1)\end{array}$ & \\
\hline & 39 & & 61 & $\begin{array}{l}\text { Имеется много шансов, что } \\
\text { информация недостоверна } \\
(\text { за }-1, \text { против }-3)\end{array}$ & $\begin{array}{l}\text { Вероятно, информация недосто- } \\
\text { верна - нет }\end{array}$ \\
\hline & $\begin{array}{c}14 \\
1 \\
\end{array}$ & $2 / 1 / 1$ & 86 & $\begin{array}{l}\text { Почти определенно, инфор- } \\
\text { мация недостоверна (за - } 1 \text {, } \\
\text { против -9) }\end{array}$ & $\begin{array}{l}\text { Почти определенно, информация } \\
\text { недостоверна - нет }\end{array}$ \\
\hline & & & & ЕЛОСТОВЕРНО & \\
\hline
\end{tabular}

Рис. 1

отсутствием этого знания, т.е. полностью описать объекты и явления реального мира невозможно, так как реальность бесконечна в каждом своем проявлении.

В социально-технических системах полнота информации - показатель $\eta \in[0,1)$, характеризующий меру ее достаточности для принятия решения. Это весьма неопределенный и относительный показатель, поскольку полнота информации оценивается исключительно по отношению к вполне определенной задаче. Учитывая сказанное, оценку полноты исходных данных будем осуществлять методом фильтрации путем сравнительного анализа использованной при принятии решения $r$ информации и «эталонной», которая с точки зрения лица, принимающего решение (ЛПР), достаточна для его принятия. Такую информацию будем представлять соответствующим морфологическим деревом (фильтром), состоящим из элементарных структур (рис. 2), которые задают морфологию соответствующих информационных рубрик $(H)$ с требуемым уровнем детализации $\left(h_{i}\right)$.

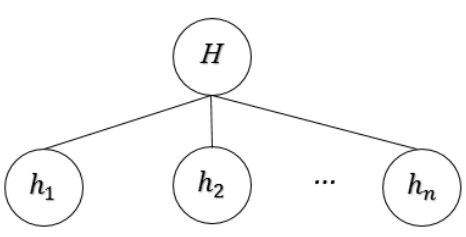

Рис. 2
Например, для принятия решения по управлению войсками в боевой операции командиру и штабу необходимо иметь, оценить и учесть различные данные обстановки. При всем многообразии эти данные группируются по составляющим боевую обстановку элементам: войска противника, свои войска, местность.

Тогда морфологическое дерево достаточных исходных данных для принятия решения командиром по управлению войсками в боевой операции может иметь следующий вид (рис. 3):

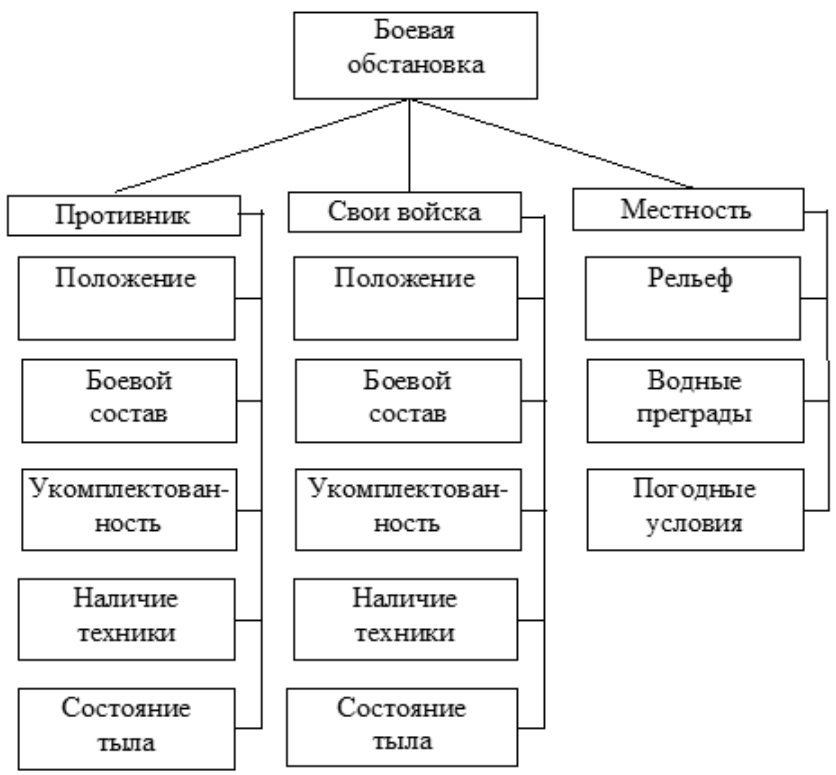

Рис. 3

Это дерево состоит из четырех элементарных морфологических структур с корневыми элементами - рубриками данных боевой обстановки и соответствующими подрубриками. Затем рубрикам (подрубрикам) этого дерева приписываются веса их влияния (важности) на элементы высшего уровня.

Далее информация, использованная при принятии решения $r$, сравнивается с морфологическим деревом путем приписывания его элементам булева параметра $\alpha_{i}: \alpha_{i}=1-$ если $i$-я руб- 
рика (подрубрика) имеется в исходных данных и $\alpha_{i}=0$ - в противном случае. И, наконец, аналогично процедуре синтеза глобальных приоритетов метода анализа иерархий, осуществляется свертка полученных оценок элементов морфологического дерева, в результате которой будет получена оценка полноты исходных данных с учетом их весомости для принятия решения.

Пусть элементы структуры (рис. 3) имеют следующие параметры: $H=(\mu, \alpha), h_{i}=\left\{\left(\mu_{i}, \alpha_{i}\right) \mid\right.$ $\mid i=\overline{1, n}\}$ где $\mu, \alpha$ и $\mu_{i}, \alpha_{i}$ - весовые коэффициенты и булевы значения элементов $H$ и $h_{i}$ соответственно. Тогда результатом свертки оценок этой структуры есть значение $a^{*}=\sum_{i=1}^{n} \mu_{i} \cdot \alpha_{i}$, которое используется в качестве параметра $\alpha$ ее родительского элемента $H$. Этот параметр, по сути, выражает степень информационной полноты соответствующей рубрики с учетом существенности ее подрубрик, и, в итоге, принимается за оценку $T(\tilde{P}(D))$ полноты исходных данных.

Отметим, что при оценке полноты данных не учитывается количественное наполнение рубрик (подрубрик), так как эти параметры обосновываются достоверностью исходных данных.

Заметим также, что весовые коэффициенты элементов морфологического дерева так же, как и веса экспертов, привлекаемые для оценки достоверности данных, можно эффективно вычислить методом анализа иерархий. При этом в условиях, когда предъявляются повышенные требования к точности результатов, для улучшения согласованности оценок парных сравнений может быть использован подход [11], в котором уменьшение рассогласованности оценок достигается при использовании их доверительных интервалов и механизма транзитивного замыкания.

Оценка качества модели выработки решения определяется степенью достоверности его положения и/или параметров управления. Под положением решения будем понимать его основную мысль (утверждение), направленную на достижение цели управления, а под параметрами управления - конкретные значения элементов объекта управления. Например, в решении «при наступлении будет задействовано 30 танков и 200 человек личного состава» положением является «при наступлении», а параметрами - «30 танков и 200 человек личного состава». В дальнейшем для простоты изложения под параметрами управления будем понимать как положения решения, так и собственно его параметры.

Достоверность параметров управления определяется тем, в какой мере модель выработки решений обеспечивает объединение формально оптимальных решений, вырабатываемых математическими моделями, и творческих замыслов человека. Пусть $p_{1}, \ldots, p_{n}$ - параметры управления решения $r$, а $\tilde{A}\left(p_{1}\right), \ldots, \tilde{A}\left(p_{n}\right)-$ нечеткие высказывания, определяющие достоверность параметров $p_{i}$ соответственно. Тогда справедлива следующая зависимость:

$$
\left(\tilde{A}\left(p_{1}\right) \wedge \ldots \wedge \tilde{A}\left(p_{n}\right)\right) \rightarrow \tilde{K}(M) .
$$

С целью получения оценки $\tilde{A}\left(p_{i}\right)$, представим процесс получения параметра $p_{i}$ функциональной операцией $\Phi \mathrm{O}_{i}$ в виде следующего кортежа:

$$
\Phi \mathrm{O}_{i}=<X_{i}, p_{i}, M_{i}>,
$$

где $X_{i}$ - входные данные, подвергаемые обработке, $p_{i}$ - результат выполнения операции, $M_{i}-$ механизм ее реализации (человек и/или компьютерная программа (математическая модель).

Механизм реализации, по сути, является моделью операции и выражает ее функциональное действие в виде отображения $M_{i}: X_{i} \rightarrow p_{i}\left(F_{i}\right)$. Если в качестве механизма выполнения операции выступает человек, то под коэффициентом $F_{i}$ понимается степень его профессионализма. В ином случае этот коэффициент определяет степень достоверности результата $p_{i}$, полученного математической моделью. Такую оценку можно также получить с помощью схемы Кента. При этом необходимо учитывать возможность математической модели получить фор- 
мально оптимальный результат $p_{i}$ на основе данных $X_{i}$.

Затем вычисляется значение истинности заключения $T\left(\tilde{A}\left(p_{i}\right)\right)$ импликации $M_{i}$. В результате $T(\tilde{K}(M))=\min _{i} T\left(\tilde{A}\left(p_{i}\right)\right)$.

Когда процесс получения параметра $p_{i}$ состоит из нескольких этапов, отображение $M_{i}$ будет многошаговым и представляется в следующем виде:

$$
\begin{gathered}
M_{i}: X_{i} \rightarrow Y_{i 1}\left(F_{i 1}\right), \\
X_{i 2} \rightarrow Y_{i 2}\left(F_{i 2}\right), \ldots, X_{i m} \rightarrow p_{i}\left(F_{i m}\right),
\end{gathered}
$$

где $X_{i j} \subseteq\left(X_{i} \cup Y_{i j-1}\right), m-$ количество этапов.

В этом случае оценка $T\left(\tilde{A}\left(p_{i}\right)\right)$ получается в результате последовательного вычисления импликаций отображения $M_{i}$.

Итак, чтобы получить оценку обоснованности управленческого решения, вначале в этом решении выделяются его элементы: информационное, организационное и оперативное решения. Затем оценивается их обоснованность и в результате определяется обоснованность управленческого решения в целом. Поскольку примеры более убедительны, чем общие рассуждения, рассмотрим один из них.

Пусть условная высота $A$ занята противником. Командиру подразделения Б поставлена задача - освободить эту высоту. Приступая к выполнению задачи, командир вначале должен оценить обстановку и решить, может ли он своими силами и средствами выполнить ее. Пусть штаб подготовил информационное решение - силами и средствами подразделения Б можно выполнить поставленную задачу. При этом предположим, что выработка решения осуществлялась на основе данных о своих войсках, войсках противника и местности.

Этап 1. Оценнка достоверности данных. Пусть исходные данные $D=\left(d_{1}, d_{2}\right)$ получены от двух источников: источник $s_{1}$ (штаб подразделения Б) - данные о своих войсках и местности $\left(d_{1}\right)$; источник $s_{2}$ (разведгруппа) - данные о войсках противника $\left(d_{2}\right)$. Далее пусть для оценки достоверности данных привлечены три эксперта $Э_{1} Э_{2}$, и $Э_{3}$, оценки которых приведены в табл. 2.

\begin{tabular}{|c|c|c|c|c|c|c|}
\hline \multirow{3}{*}{ Степень достоверности данных } & \multicolumn{6}{|c|}{ Данные } \\
\hline & \multicolumn{3}{|c|}{$d_{1}$} & \multicolumn{3}{|c|}{$d_{2}$} \\
\hline & $Э_{1}$ & $Э_{2}$ & $Э_{3}$ & $Э_{1}$ & $Э_{2}$ & $Э_{3}$ \\
\hline Данные достоверны (99\%) & + & + & + & & & \\
\hline $\begin{array}{l}\text { Вероятно, данные достоверны } \\
(75 \%)\end{array}$ & & & & + & & + \\
\hline $\begin{array}{l}\text { Данные достоверны в той же } \\
\text { мере, что и недостоверны (50\%) }\end{array}$ & & & & & + & \\
\hline $\begin{array}{l}\text { Вероятно, данные } \\
\text { недостоверны }(25 \%)\end{array}$ & & & & & & \\
\hline Данные недостоверны (1\%) & & & & & & \\
\hline
\end{tabular}

Т а бли ц а 2

В табл. 2 приведена возможная количественная интерпретация степеней достоверности в схеме Кента.

Используя данные оценки достоверности данных по формуле (6), с учетом весовых коэффициентов экспертов 0,4; 0,3 и 0,3, получим: $p\left(d_{1}\right)=0,99 ; p\left(d_{2}\right)=0,675$ и оценку достоверности исходных данных в целом $T(\tilde{A}(D))=$ $=0,675$.

Этап 2. Оценка полноты данных. Для оценки полноты данных используем морфологическое дерево (рис. 3). Пусть веса рубрик (подрубрик) имеют следующие значения: для рубрик $-0,5 ; 0,4 ; 0,1$, а для их подрубрик соответственно $(0,1 ; 0,3 ; 0,2 ; 0,2 ; 0,2),(0,1 ; 0,3 ; 0,2$; $0,2 ; 0,2)$ и $(0,4 ; 0,4 ; 0,2)$.

Далее пусть $(1,1,1),(1,1,0,1,0),(1,1,1,1,1)$ и $(1,1,1)$ - булевы параметры рубрик и соответствующих подрубрик этого морфологического дерева. Тогда в результате свертки оценок его элементов получим следующие значения параметров полноты: для рубрик - 0,6; 1,0 и 1,0; для исходных данных в целом - 0,8, т.е. $T(\tilde{P}(D))=0,8$.

Этап 3. Оиенка качества модели выработки решения. Данное решение содержит только одно положение - силами и средствами подразделения Б можно выполнить поставленную задачу. Пусть процесс выработки этого положения (решения) состоит из двух функцио- 
нальных операций: $\Phi \mathrm{O}_{1}$ - расчет соотношения сил и средств противоборствующих сторон, $\Phi \mathrm{O}_{2}$ - анализ полученных результатов и формулировка решения.

Представим первую операцию в виде $\Phi \mathrm{O}_{1}=<X_{1}, p_{1}, M_{1}>$, где $X_{1}-$ данные о своих войсках и войсках противника, $p_{1}$ - соотношение сил и средств противоборствующих сторон, скажем, 3:1 (боевой потенциал подразделения Б в три раза превышает потенциал противника), $M_{1}: X_{1} \rightarrow p_{1}\left(F_{1}=1\right)$ - операция реализуется математической моделью. Поскольку $T\left(X_{1}\right)=0,675$, то $T\left(\tilde{A}\left(p_{1}\right)\right)=0,675$.

Аналогично $\Phi \mathrm{O}_{2}=<X_{2}, p_{2}, M_{2}>$. Здесь $X_{2}$ - соотношение 3:1 и данные о местности, $p_{2}-$ формулировка решения, $M_{2}: X_{2} \rightarrow p_{2}\left(F_{2}=0,9\right)$ - операция реализуется человеком (например, начальником штаба подразделения Б), который с учетом полученного соотношения сил и средств, характеристик местности и норм руководящих документов по ведению боевых действий, формулирует соответствующее решение. Входными данными этой операции является результат выполнения предыдущей, т.е. $T\left(X_{2}\right)=0,675$. Поэтому $T\left(\tilde{A}\left(p_{2}\right)\right)=0,675$ и в результате имеем $T(\tilde{K}(M))=0,675$. Тогда в целом, используя (5), получим $T(\tilde{O}(r))=0,675$, т.е. обоснованность предложенного штабом решения по шкале Харрингтона высока.

Заметим, что поскольку окончательное решение принимает человек и несет полную ответственность за последствия от его реализации, поэтому ЛПР, кроме оценки обоснованности выработанного решения также желательна, а в некоторых случаях необходима информация, на основе которой была получена такая оценка, т.е. информация о достоверности и полноте исходных данных, что позволит либо придать ему уверенность и развеять сомнения в целесообразности принятия этого решения, либо отправить его на доработку. Поэтому командиру подразделения Б представляется следующая информация (табл. 3).
Т а б ли ц а 3

\begin{tabular}{|l|c|l|}
\hline \multirow{2}{*}{\multicolumn{1}{|c|}{ Информация }} & \multicolumn{2}{|c|}{ Оценка } \\
\cline { 2 - 3 } & количественная & лингвистическая \\
\hline Обоснованность решения & 0,675 & \multicolumn{1}{|c|}{ высокая } \\
\hline Достоверность данных: & & вероятно досто- \\
войска противника & 0,675 & верны \\
свои войска & 0,99 & достоверны \\
местность & 0,99 & достоверны \\
\hline Полнота данных: & 0,8 & полны на $80 \%$ \\
войска противника & 1,0 & полны \\
свои войска & 1,0 & полны \\
местность & \multicolumn{2}{|l}{} \\
\hline
\end{tabular}

Заключение. Рассмотренный подход к оценке обоснованности управленческих решений не претендует на завершенность и служит лишь иллюстрацией применения основных идей нечеткой логики для получения такой оценки. Учет в данном подходе морфологии решений и факторов, определяющих их обоснованность, причинно-следственных связей между ними и экспертных суждений позволяет доступным путем получить доказательную оценку обоснованности. Предлагаемый подход может быть использован в качестве пилотажа для разработки соответствующих методик обоснования принимаемых управленческих решений в различных сферах деятельности.

1. Герасимов Б.М., Самохвалов Ю.Я. Методы оценки обоснованности решений в интеллектуальных системах // Сучасні інформаційні технології у сфері безпеки та оборони, 2009, №2(5), С. 9-12.

2. Матвеева И.Г., Якушев А.А. Теория и практика современных методов обоснования управленческих решений // Экономика и современный менеджмент: теория и практика: Сб. ст. XXIX междунар. науч.практ. конф., Новосибирск: СибАК, 2013, № 9(29), C. 52-61.

3. Шейгас А.К., Афанасьев В.В. Оценка обоснованности принятого решения при назначении воздействий авиацией по объектам противника // Системи обробки інформації, 2014, № 7(123), С. 172-174.

4. Вешнева И.В. Математические модели в системе управления качеством высшего образования с использованием методов нечеткой логики. - Саратов: Саратовский источник, 2010, 187 с.

5. Гупанова Ю.Е. Применение инструментария нечеткой логики в оценке качества таможенных услуг // Экономический анализ: теория и практика. М.: Финансы и кредит, 2016, № 1(448), Р. 143-158.

6. Лялькова E.E. Информационные источники управленческого анализа // Управление экономическими системами, 2016, № 8(90), С. 25. 
7. Information quality evaluation in fusion systems, in Information Fusion / I.-G. Todoran, L. Lecornu, A. Khenchaf et al., 2013, P. 906-913.

8. Kent $S$. Strategic Intelligence for American World Policy. Princeton: Princeton Univ. Press, 1949, 226 p.

9. Плэтm $B$. Информационная работа стратегической разведки. Основные принципы. М.: ИИЛ, 1958. 144 с.
10. Глушков В.М. О прогнозировании на основе экспертных оценок // Кибернетика, 1969, № 2, С. 8-17.

11. Самохвалов Ю.Я. Согласование экспертных оценок в матрицах отношений предпочтения // УСиМ, 2002, № 6, C.49-53.

Поступила 07.03.2017

E-mail:yu1953@ukr.ua

(C) Ю.Я. Самохвалов, 2017

UDC 681.3.08

Yu.Ya. Samokhvalov ${ }^{1}$

\title{
The Assessment of the Administrative Decisions Validity by Fuzzy Logic
}

\author{
${ }^{1}$ Doctor of Technical Sciences, Professor of the Department of cyber security and information protection of Taras Shevchenko \\ National University of Kyiv, Volodymyrska, 64, yu1953@ukr.ua
}

Keywords: administrative decision, quality and validity of decisions, ambiguity, linguistic variable, fuzzy logic, proof.

Introduction. The decision is the basis of any control. The validity defines the quality of decisions, and it is impossible to measure it in the process of management. About the quality of the adopted and implemented decisions, validity can be judged only by its consequences. Therefore, a priori assessment of the reasonableness of the solutions is essential.

Purpose. There are several approaches to evaluating the solutions validity: variational, based on the criterion of efficiency, probabilistic and informational. In these methods, the accuracy of the solutions is estimated by the deviations of control parameters from the extreme values inherent in optimal solutions, which leads to a knowledge of this decision and, as a rule, the absence of such knowledge. Besides, when evaluating the solutions reasonableness, the characteristics of the process of their production are taken into account, which does not always provide the reliability of such estimates. In this regard, one possible approach to assessing the validity of decisions is proposed based on the logical-linguistic analysis of the solutions morphology, factors determining the quality and impact of these factors on the decisions validity, that determines its purpose and content.

Methods. The expert assessment and fuzzy logic mechanisms are used.

Results. The term validity is considered as the fuzzy variable with the Zadeh membership function, which connects the decision validity with its rationality. The factors that determine the validity of the decisions are defined. The procedures for quantitative estimation of the truth of these factors and the truthfulness of the solution using the compositional rule of the fuzzy modus ponens are created. The scale of approximation of the quantitative values of the validity of the linguistic modal assessments are designed.

Conclusion. This approach for assessing the validity of administrative decisions does not claim to completeness and is used only to illustrate the application of the fundamental ideas of fuzzy logic for this evaluation. Accounting in this approach, the morphology of the decisions and factors determining their validity, the causal relationships between them and expert judgment allows you to get a final assessment of the reasonableness. The proposed approach can be using as a pilot project for the development of appropriate methods of substantiation of managerial decisions in various fields.

1. Gerasimov V.M., Samokhvalov Yu.Ya. Methods of assessing the validity of decisions in intelligent systems // Modern information technologies in the sphere of security and defence, 2009, № 2(5), C. 9-12.

2. Matveeva I.G., Yakushev A.A.. Theory and practice of modern methods of management decisions // Economics and modern of management: theory and practice: a collection of stata XXIX Intern. Scientific-practical conference, Novosibirsk: SibAK, 2013. - № 9(29).

3. Chagas A.K., Afanasiev V.V. Assessment of the reasonableness of the decision in the appointment of the impacts of aviation on the enemy objects // Systems pf information processing, 2014, № 7(123), C. 172-174.

4. Vasneva I.V. Mathematical models in the quality management system of higher education using the methods of fuzzy logic, Saratov: Saratov sourse, 2010, 187 c.

5. Gupanova Yu.Ye. Application of Fuzzy logic in the assessment of the quality of customs services // Economic analysis: theory and practice, M.: Finances and credit, 2016, № 1(448), P. 143-158.

6. Lyalkova Ye.Ye. Information sources of management analysis // Management of economic systems: Electronic scientific journal, Kislovodsk Institute of Economics and Law, 2016, № 8(90), 2016, C. 25.

7. Information quality evaluation in fusion systems, in Information Fusion / I.-G. Todoran, L. Lecornu, A. Khenchaf et al., 2013, P. 906-913.

8. Kent S. Strategic Intelligence for American World Policy. Princeton: Princeton Univ. Press, 1949, 226 p.

9. Plett $V$. Information work of strategic intelligence. Basic principles. Foreign Moscow: Literature Publishing House, 1958, 144 p.

10. Hlushkov V.M. On the prediction based on expert estimates // Kibernetika, 1969, № 2, C. 8-17.

11. Samokhvalov Yu.Ya. Harmonization of expert assessments in matrices of reference relations // Upravlyayushchie Sistemy i Mashiny, 2002, № 6, P. 49-53. 